

\title{
EVALUACIÓN DEL MEJORAMIENTO DEL CONFORT TÉRMICO CON LA INCORPORACIÓN DE MATERIALES SOSTENIBLES EN VIVIENDAS EN AUTOCONSTRUCCIÓN EN BOSA, BOGOTÁ, COLOMBIA
}

\author{
AN EVALUATION OF THE IMPROVEMENT OF THERMAL COMFORT \\ WITH THE INCORPORATION OF SUSTAINABLE MATERIALS IN SELF- \\ BUILD DWELLINGS IN BOSA, BOGOTA, COLOMBIA.
}

\author{
FRANZ CALDERON URIBE \\ Doctor en Comunicación visual en Arquitectura y Diseño \\ Investigador Asociado \\ Universitaria Agustiniana, Uniagustiniana, Colombia \\ https://orcid.org/0000-0002-3008-7552 \\ franz.calderon@uniagustiniana.edu.co
}

\section{RESUMEN}

En este artículo se evalúa cómo la incorporación de materiales sostenibles mejoró el confort térmico en un hábitat en proceso de autoconstrucción, en el barrio San José de Bosa, en Bogotá, Colombia. El problema del confort se ha abordado como una condición fundamental del hábitat que responde a una necesidad de cobijo, de tener un espacio propio, sin importar la forma en que este espacio responda a las variables climáticas. Por otra parte, el uso de materiales sostenibles ha ido incorporando beneficios ambientales, en especial en las grandes ciudades, debido a los altos índices de contaminación actuales. En los barrios periféricos, como el aquí abordado, el cemento, el ladrillo y el acero son los materiales más usados en la construcción. Concretamente, se expone en lo que sigue un estudio de caso en el que se hicieron mediciones periódicas con el objetivo de determinar el balance térmico y el modo en que los materiales empleados respondían a las condiciones climáticas existentes. Con el fin de optimizar no solo el confort térmico sino la sostenibilidad del hábitat, se incorporaron materiales sostenibles, para luego realizarse nuevas mediciones, a partir de las cuales se determinó que los nuevos materiales mejoraron el confort térmico del hábitat, al comprobarse un aumento de $6^{\circ} \mathrm{C}$ en la temperatura media interior.

Palabras clave

hábitat, materiales alternativos, diseño sustentable, balance térmico

\section{ABSTRACT}

This article evaluates how the incorporation of sustainable materials improved thermal comfort in a habitat using the self-build process, in the San José de Bosa neighborhood in Bogota, Colombia. The problem of comfort was addressed as a fundamental condition of the habitat. The habitat responds to a need for shelter, understood as the need to have one's own space regardless of the way this space responds to climate variables. The use of sustainable materials provides environmental benefits, especially for large cities due to the current high levels of pollution (Fernández-Agüera, DomínguezAmarillo, Alonso, \& Martín-Consuegra, 2019). In these peripheral neighborhoods, cement, brick and steel are the most commonly used construction materials. A case study was carried out in which periodic measurements were made with the objective of determining the thermal balance and how the materials used responded to the existing climate conditions. In order to improve not only the thermal comfort but also the sustainability of the habitat, sustainable materials were used. Finally, new measurements were made to determine if the new materials improved the thermal comfort of the habitat and an increase of $6{ }^{\circ} \mathrm{C}$ in the average indoor temperature was established. 


\section{INTRODUCCIÓN}

Este artículo es el resultado de un proyecto de investigación académico cuyo objetivo principal es el análisis térmico de un hábitat informal en proceso de autoconstrucción ubicado en Bosa, un distrito popular situado al sur occidente de Bogotá. El estudio determinó que, con la incorporación de una solución constructiva sostenible, es posible optimizar el confort térmico en un hábitat autoconstruido. El análisis térmico en esta tipología de vivienda informal no ha sido estudiado en Colombia, sin embargo hay antecedentes en este tema en otras ciudades latinoamericanas, como la de Guayaquil (Ecuador) donde se han llevado a cabo estudios de casos que revelaron que los materiales empleados, especialmente en la cubierta, no generaban confort térmico (Macias, Soriano, Sanchez y Canchingre, 2015).

El análisis comparativo de diferentes trabajos e investigaciones pudo determinar que en los países desarrollados se dan algunos factores diferenciadores: existe una normativa muy clara que obliga a las empresas constructoras a cumplir con unos estándares mínimos de calidad, sobre todo en torno a los materiales empleados, además de tener parámetros de evaluación del confort térmico y acústico. En algunos países en vías de desarrollo latinoamericanos se ha evidenciado que el hábitat popular y autoconstruido no tiene en cuenta aspectos bioclimáticos ni referidos al confort térmico, y que los materiales empleados son reutilizados o temporales, debido a la informalidad del hábitat construido.

El proyecto evaluó el mejoramiento del confort térmico en un hábitat autoconstruido, con materiales tradicionales, cemento, ladrillo, materiales reutilizados, tejas de zinc, tejas de asbesto cemento y elementos de madera, entre otros. Se reemplazaron los materiales existentes por elementos constructivos obtenidos a partir del reciclaje de envases de Tetrapak y aislamiento de origen vegetal. La investigación pudo determinar que el elemento constructivo que más afectaba el confort térmico del hábitat era la cubierta, por esta razón esta se reemplazó incorporando materiales sostenibles que fortalecieran el aislamiento térmico del hábitat $y$, por ende, aumentaran el confort térmico del interior del hábitat objeto de estudio.

\section{ESTADO DEL ARTE.}

El confort térmico se define en la Norma ISO 7730 como "esa condición de la mente en la que se expresa la satisfacción con el ambiente térmico"(Robledo-Fava et al., 2019)we analyze the influence of the designer's choice of values for the human metabolic index (met. Si bien esta definición se encuentra aceptada ampliamente, es difícil su traducción a parámetros físicos cuantificables. Básicamente, y en términos generales, el hombre califica un ambiente como confortable cuando ningún tipo de incomodidad térmica está presente. La primera condición de confort es la neutralidad térmica, lo que significa que la persona no se siente demasiado calurosa, ni demasiado fría (Godoy Muñoz, 2012).

En los últimos 30 años este concepto ha tomado un carácter muy relevante en el diseño y construcción de viviendas, producto de los avances de la ingeniería, de los sistemas de aire acondicionado y la climatización. Las diversas implicaciones sociales y de consumo energético que ello conlleva obligan a analizar y reinterpretar los modos tradicionales de vida en regiones con climas tropicales, y a buscar soluciones relacionadas directamente la arquitectura bioclimática. Por ejemplo, los edificios ventilados naturalmente no solo dan sensación de confort a los ocupantes y consumen menos energía, sino también están vinculados con su cultura, con una manera particular de entender la relación interior-exterior e, incluso, la privacidad (Godoy Muñoz, 2012).

Además de los aspectos bioclimáticos, es importante mencionar las teorías de Fanger, basadas en experimentos realizados a 1296 jóvenes en cámaras térmicas, en los que se usó modelos estáticos de transferencia de calor. En estos estudios, se analizó la ropa y la actividad de los participantes, mientras eran expuestos a diferentes ambientes térmicos. Los participantes debían indicar cómo se sentían en relación al calor y el frío, usando los siete puntos de la escala de sensación térmica de ASHRAE, de (-3) hasta (+3), que considera solo los valores enteros y donde los valores negativos representan la sensación de frío; los positivos, la de calor; y el valor 0 , la sensación térmica neutral. Otra parte del estudio consistió en que los participantes controlaran las condiciones térmicas ambientales, ajustando estas hasta que se sintieran en confort. El modelo de Fanger combina las teorías de balance térmico con la fisiología y la termorregulación para determinar un rango de temperaturas de confort, en las cuales los ocupantes del edificio se sienten bien. De acuerdo con estas teorías, el cuerpo humano emplea procesos fisiológicos como sudoración, temblor y vasodilatación, con el objetivo de mantener el balance térmico entre el calor producido por el metabolismo y la pérdida de calor a través del cuerpo. Mantener este balance de calor es la primera condición para conseguir una sensación térmica neutral. Sin embargo, Fanger observó que el sistema termorregulador del hombre es tan eficiente que es capaz de crear equilibrio térmico dentro de amplios límites de variables ambientales, incluso aunque no exista confort (Godoy Muñoz, 2012).

Para ser capaz de predecir las condiciones en las que tiene lugar la neutralidad térmica, Fanger investigó los procesos fisiológicos del cuerpo que se suceden cerca de la neutralidad. Finalmente, determinó que los únicos procesos fisiológicos que influyen en el balance térmico en este contexto son: la temperatura media de la piel y la tasa de sudoración, procesos que están en función del nivel de actividad. Con posteriores investigaciones, Fanger obtuvo una relación lineal, justamente, entre nivel de actividad y tasa de sudoración. Otra investigación, donde 
participantes con una ropa estándar tomaban parte de una serie de pruebas dentro de una cámara térmica mientras realizaban cuatro niveles de actividad diferentes, sirvió para evidenciar una relación lineal entre nivel de actividad y temperatura de la piel. Tras sustituir estos dos valores por sus respectivas regresiones lineales en la ecuación de balance térmico, Fanger obtuvo su ecuación de confort, la cual predice las condiciones necesarias para que el ocupante sienta neutralidad térmica (Macias et al., 2015).

El análisis del confort térmico constituye un tema recurrente en los estudios de caso y los análisis de consumo de energía asociados a la sostenibilidad de una construcción. En los últimos cinco años se advirtió que los países en vías de desarrollo están empezando a incorporar conceptos de sostenibilidad asociaos a la resolución de problemas típicos de la región, como los sismos y el uso de materiales reciclados (Sekhar y Nayak, 2018). Un ejemplo de esta nueva tendencia es el desarrollo de un suelo construido sobre neumáticos reciclados que absorben los movimientos producidos por un terremoto, destinado a la construcción de hábitats en Ecuador.

Tradicionalmente, estos estudios se centraban en medir los espacios interior y exterior con el fin de determinar las características físicas de los materiales, consumos de energía relacionados con la normativa del país en el que se hiciera la investigación. Hoy, existe una creciente preocupación en Europa occidental de que un mayor aislamiento y estanqueidad de los edificios residenciales aumenta el riesgo de sobrecalentamiento. En tal sentido, Jones, Goodhew y de Wilde (2016) efectuaron el monitoreo de la temperatura de dos casas idénticas en el suroeste del Reino Unido que fueron construidas con bajos estándares de energía (Código para hogares sostenibles Nivel 5). Los datos de temperatura se examinaron utilizando los criterios establecidos de sobrecalentamiento estático (Guía CIBSE A) y un estándar de confort térmico adaptativo (BSEN15251). Se encontró que las casas pueden considerarse incómodamente cálidas durante el verano y corren el riesgo de sobrecalentarse. El estudio sugirió que el comportamiento de los ocupantes juega un papel importante en la reducción o aumento de las temperaturas internas.

Así también, se ha asociado el confort térmico al consumo de recursos naturales el cual afecta el medio ambiente de la región respectiva. Eso ocurrió en el estado de Jammu y Cachemira en la India, que experimentó una grave crisis energética debido a la baja disponibilidad de energía per cápita. Tal situación obligó a la población a un extensivo consumo de madera, que condujo a la deforestación del entorno, por lo que fue obligatorio avanzar hacia una estrategia energética sostenible completa. Un estudio (Ahmed, Qayoum y Mir, 2019) pudo determinar que un eficiente y sostenible aislamiento térmico de los edificios era una gran oportunidad para generar e incentivar el ahorro de energía. Este se centró en el uso de nuevos materiales aislantes que incorporan componentes reciclados y sostenibles. El uso constante de materiales aislantes en los edificios, no solo redujo el consumo de energía, sino que además redujo la emisión de gases de efecto invernadero y proporcionó un mejor confort térmico interior. De esta forma, se empleó aislantes naturales como lana de oveja, lana de cabra y crin de caballo, que se procesan y caracterizan mejor en términos de absorción de humedad, conductividad térmica, análisis termo-gravimétrico (TGA) y calorimetría diferencial de barrido (DSC).

Entre otro de los trabajos revisados destaca el de Fernández, Domínguez, Alonso y Martín (2019), sobre confort térmico y calidad del aire interior (IAQ) en edificios residenciales con diferentes grados de hermeticidad, en dos climas en España. El proyecto comparó el comportamiento de las áreas ocupadas de día y las de noche. El IAQ de los edificios estudiados, erigidos antes de que las regulaciones de eficiencia energética estuvieran vigentes (1939-1979) y carentes de ventilación mecánica, se comparó con su estanqueidad. La razón de ese enfoque fue que, en tales circunstancias, el cambio de aire depende de una ventilación natural no controlada (= abrir ventanas), en consecuencia, de la temperatura exterior. La humedad relativa también se tuvo en cuenta, dada la condensación que puede inducirse cuando la ventilación es insuficiente. Finalmente, se pudo determinar que, en invierno, en ambos climas, los niveles de $\mathrm{CO}^{2}$ fueron superiores a 1200 ppm, con promedios del orden de 1900 ppm en Madrid y 1400 ppm en Sevilla, y más altos durante la noche que durante el día. Los cambios de aire cada hora, mediados por la infiltración, parecían ser insuficientes para mantener la casa en condiciones saludables, además de presentarse un alto riesgo por condensación superficial en las viviendas más herméticas. Es decir, y a pesar de tener ventilación forzada, los edificios necesitaban de una ventilación natural.

Por último, cabe mencionar la investigación de Chowdhury y Neogi (2019), cuyo propósito fue analizar el rendimiento térmico de paredes y techos, de uso común en el sector de la construcción residencial en la India. Esto se llevó a cabo mediante la determinación del coeficiente global de transferencia de calor o el valor $U$, utilizando la instalación de prueba de caja caliente protegida. El procedimiento de prueba general estuvo de acuerdo con BS EN ISO 8990: 1996. Posteriormente, se estudió el efecto de la variación de la temperatura diferencial del aire sobre el coeficiente global de transferencia de calor del techo de concreto de cemento reforzado y tres tipos de tipologías usados en la construcción de paredes de ladrillo hechos en arcilla cocida. A partir del análisis de regresión, se pudo evaluar fácilmente el valor $U$ de las paredes y el techo para cualquier temperatura diferencial, según lo experimentado por los edificios en varias zonas climáticas en la India. Asimismo, fue posible determinar que los edificios proporcionaban confort térmico a través de la interacción entre el ambiente externo y los elementos constructivos que lo conforman, paredes, techos y acristalamientos. Un buen reconocimiento y análisis previo del rendimiento térmico de los materiales empleados en paredes, techos 
y acristalamientos puede, en definitiva, ayudar a estimar las cargas de calefacción o refrigeración en los sistemas de ventilación y aire acondicionado propuestos.

\section{METODOLOGÍA}

El presente proyecto expone un estudio de caso, en el cual se emplearon dos métodos: el primero, definido por el protocolo dado por la norma ISO 7730, corresponde al análisis de aspectos climatológicos del entorno, habitual en este tipo de estudios (temperatura, humedad, velocidad del viento, entre otros); y el segundo, a una caracterización de los materiales existentes que tiene como objetivo evaluar su inercia térmica a través de herramientas estáticas de análisis de confort térmico (figura de Mahoney y diagrama de Givoni).

La norma ISO 7730 Método Fanger establece que no solo el balance térmico es necesario en un espacio para tener confort térmico; además de tener una temperatura adecuada, se debe tener en cuenta:

a. Las características del vestuario: aislamiento y área total del mismo.

b. Las características del tipo de trabajo: carga térmica metabólica y velocidad del aire.

c. Las características del ambiente: temperatura seca, temperatura radiante media, presión parcial del vapor de agua en el aire y velocidad del aire.

Tomando como punto de partida el modelo, se establecieron las siguientes características:

\section{a) Características del vestuario.}

En este caso, se determinó un aislamiento medio vestuario completo, 1.0 clo. (clothig). Unidad térmica equivalente una resistencia térmica de $0,18 \mathrm{~m}^{2} \mathrm{hr}{ }^{\circ} \mathrm{C} / \mathrm{Kcal}$ (Castillo y Huelsz, 2017)-

Aquí, los usuarios del hábitat analizado llevaban ropa para protegerse del frío de la zona.

\section{b) Características del trabajo.}

Considerando la carga térmica metabólica y la velocidad del aire, se usó la figura establecida por la norma UNE ISO 7726:2002 que determina este valor a partir de las características del vestuario, la temperatura media y la velocidad del aire (Figura 4).

\section{c) Características del ambiente.}

En esta fase de la investigación se recopiló toda la información del entorno del hábitat analizado, la cual luego se comparó con los datos obtenidos en el interior del hábitat. Para ello se emplearon dos equipos que midieron directamente el índice térmico WBGT (Wet Bulb Globe Temperature), además de la temperatura, humedad y temperatura del globo negro. El instrumento empleado fue el modelo HT30 Extech: un medidor de la velocidad del aire y la humedad (Figura 1).

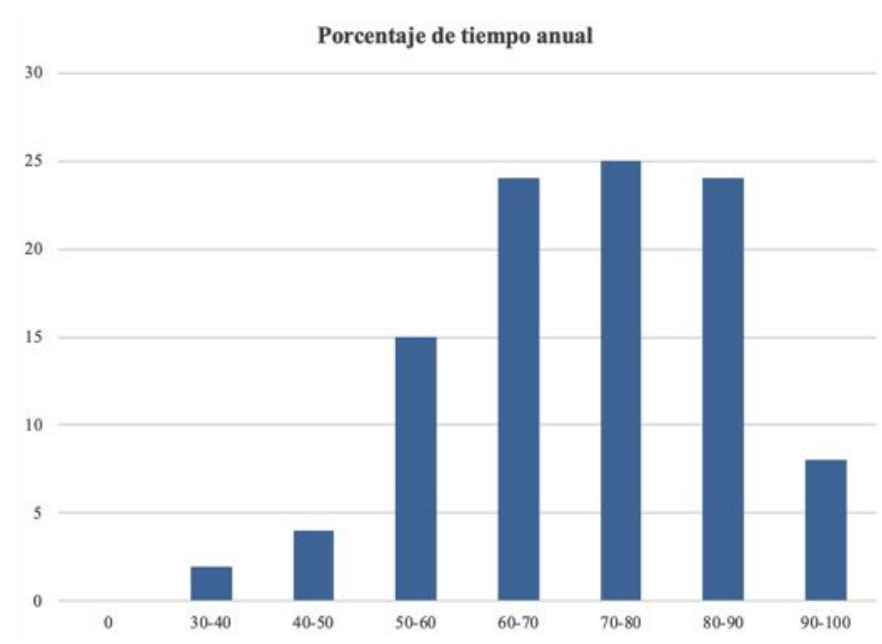

Figura 1. Temperatura del aire. Fuente: Elaboración del autor.

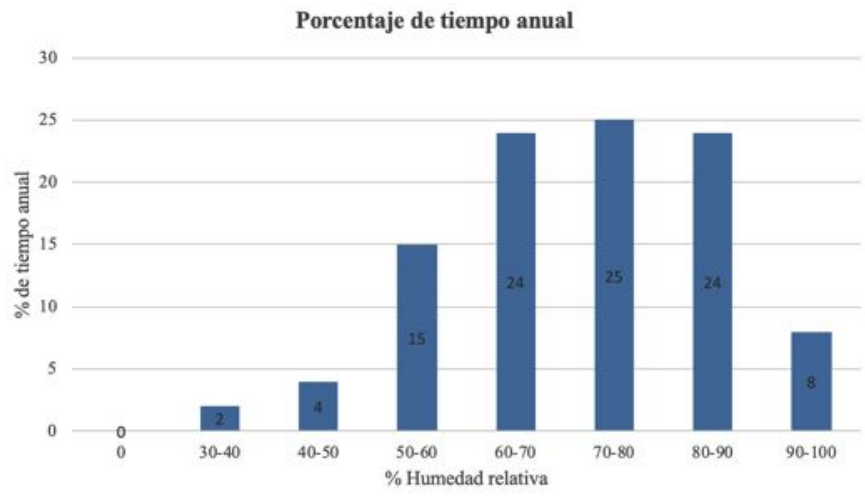

Figura 2. Humedad relativa. Fuente: Elaboración del autor.

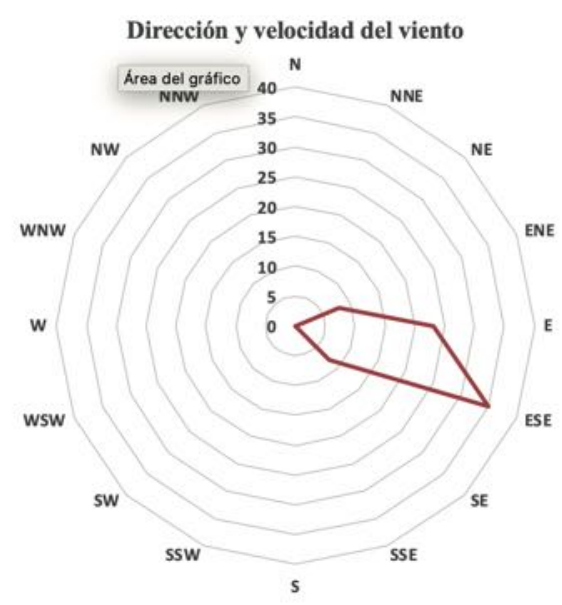

Figura 3. Rosa de los vientos de la zona. Fuente: Elaboración del autor.

Estos son los valores que se tomaron para el cálculo: Tg: 17.7 ; Ta:16.2 ; Va:0.20 m/s ; Hr:67\% ; M:1.2met. 


\begin{tabular}{|c|c|c|c|c|c|c|c|c|c|}
\hline \multirow[t]{2}{*}{$\begin{array}{l}\text { Vestuario } \\
\text { Clo }\end{array}$} & \multirow{2}{*}{$\begin{array}{c}\text { Temperatura } \\
\text { Operativa } \\
{ }^{\circ} \mathrm{C}\end{array}$} & \multicolumn{8}{|c|}{$\begin{array}{l}\text { Velocidad relativa del aire } \\
\mathrm{m} / \mathrm{s}\end{array}$} \\
\hline & & $<0,10$ & 0,10 & 0,15 & 0,20 & 0,30 & 0,40 & 0,50 & 1,0 \\
\hline \multirow{8}{*}{0,5} & 18 & $-2,01$ & $-2,01$ & $-2,17$ & $-2,38$ & $-2,70$ & & & \\
\hline & 20 & $-1,41$ & $-1,41$ & $-1,58$ & $-1,76$ & $-2,04$ & $-2,25$ & $-2,42$ & \\
\hline & 22 & $-0,79$ & $-0,79$ & $-0,97$ & $-1,13$ & $-1,36$ & $-1,54$ & $-1,69$ & $-2,17$ \\
\hline & 24 & $-0,17$ & $-0,20$ & $-0,36$ & $-0,48$ & $-0,68$ & $-0,83$ & $-0,95$ & $-1,35$ \\
\hline & 26 & 0,44 & 0,39 & 0,26 & 0,16 & $-0,01$ & $-0,11$ & $-0,21$ & $-0,52$ \\
\hline & 28 & 1,05 & 0,98 & 0,88 & 0,81 & 0,70 & 0,61 & 0,54 & $-0,31$ \\
\hline & 30 & 1,64 & 1,57 & 1,51 & 1,46 & 1,39 & 1,33 & 1,29 & 1,14 \\
\hline & 32 & 2,25 & 2,20 & 2,17 & 2,15 & 2,11 & 2,09 & 2,07 & 1,99 \\
\hline \multirow{8}{*}{1} & 16 & $-1,18$ & $-1,18$ & $-1,31$ & $-1,43$ & $-1,59$ & $-1,72$ & $-1,82$ & $-2,12$ \\
\hline & 18 & $-0,75$ & $-0,75$ & $-0,88$ & $-0,98$ & $-1,13$ & $-1,24$ & $-1,33$ & $-1,59$ \\
\hline & 20 & $-0,32$ & $-0,33$ & $-0,45$ & $-0,54$ & $-0,67$ & $-0,76$ & $-0,83$ & $-1,07$ \\
\hline & 22 & 0,13 & 0,10 & 0,00 & $-0,07$ & $-0,18$ & $-0,26$ & $-0,32$ & $-0,52$ \\
\hline & 24 & 0,58 & 0,54 & 0,46 & 0,40 & 0,31 & 0,24 & 0,19 & 0,02 \\
\hline & 26 & 1,03 & 0,98 & 0,91 & 0,86 & 0,79 & 0,74 & 0,70 & 0,58 \\
\hline & 28 & 1,47 & 1,42 & 1,37 & 1,34 & 1,28 & 1,24 & 1,21 & 1,12 \\
\hline & 30 & 1,91 & 1,86 & 1,83 & 1,81 & 1,78 & 1,75 & 1,73 & 1,67 \\
\hline
\end{tabular}

Figura 4. Nivel de actividad 1.2 met. Fuente: Elaboración del autor.

Las mediciones se realizaron entre las 14:00 y las 05:00 horas, de forma periódica, una vez a la semana, durante los meses de agosto, septiembre y octubre, y se compararon los datos exteriores con los datos del interior del hábitat (Figura 7). Es necesario aclarar que, para el cálculo de la temperatura operativa, en este caso, solo se tuvieron en cuenta las temperaturas diurnas ya que las nocturnas están fuera de los rangos establecidos por la norma (Figura 4).

Las mediciones nocturnas permitieron determinar que el hábitat no presenta confort térmico a partir de las 21:00 horas, debido a las bajas temperaturas exteriores; el hábitat analizado no tiene materiales que mantengan esta temperatura operativa.

Antes de determinar la temperatura operativa es necesario calcular la temperatura radiante media:

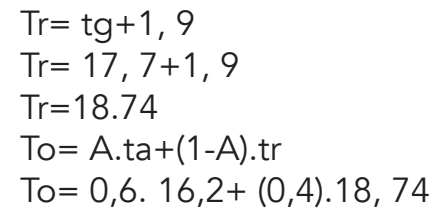

$\mathrm{To}=17,21^{\circ} \mathrm{C}$

\section{ANÁLISIS DEL CORRESPONDIENTE EVALUADAS \\ BALANCE TÉRMICO A LAS CONDICIONES}

La zona geográfica estudiada fue el distrito de Bosa en el sur de Bogotá, la que presenta variaciones de temperatura entre el día en la noche: durante el día la temperatura promedio ronda los índices de confort térmico (18-20 grados centígrados) (IDEAM, 2007). Con estas temperaturas los materiales empleados hasta ahora funcionarían correctamente, sin embargo, en la noche y la madrugada las temperaturas mínimas son de 7.6 grados centígrados y la humedad relativa llega al $70 \%$, incrementando la sensación térmica de frío. A partir de estas mediciones se emplearon dos herramientas tradicionales en este tipo de análisis: la Figura de Mahoney y el gráfico de Givoni (Figuras 5 y 6$)$.

En la figura de Mahoney se especifica que los muros y la cubierta deben tener materiales con una alta inercia térmica (en el momento de hacer el análisis, la cubierta presentó una baja inercia térmica) y que el hábitat debería poseer unas aperturas de entre 
Evaluación del mejoramiento del confort térmico con la incorporación de materiales sostenibles en viviendas en autoconstrucción en Bosa, Bogotá

Franz Calderon Uribe

ESTRÉS TÉRMICO

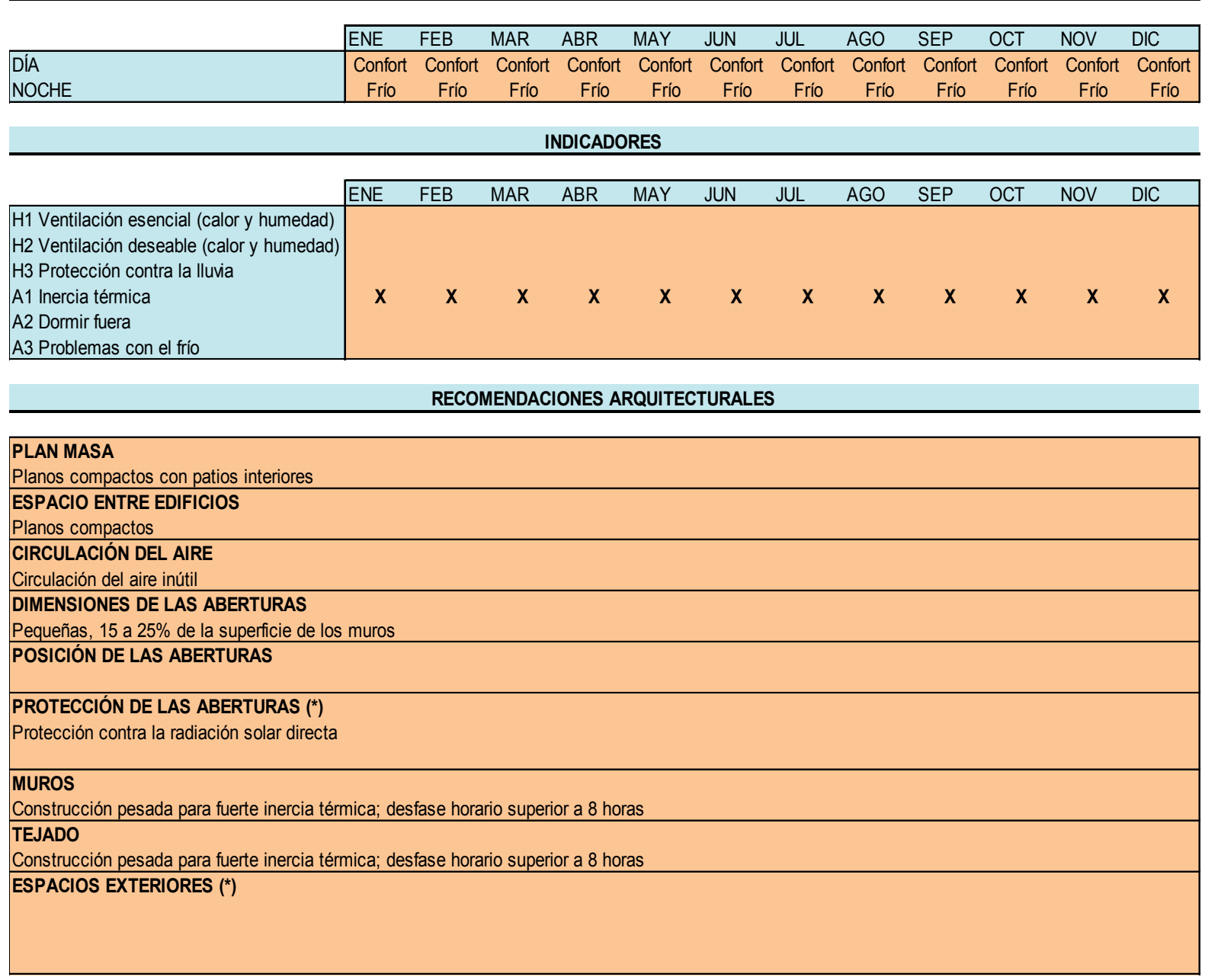

Figura 5. Figura de Mahoney para la zona de Bosa. Fuente: Elaboración del autor.

GRÁFICO PSICOMÉTRICO DE CONFORT - URBANO

CARTA DE GIVONI

Gráfico aplicable a la ciudad de Bogotá Colombia
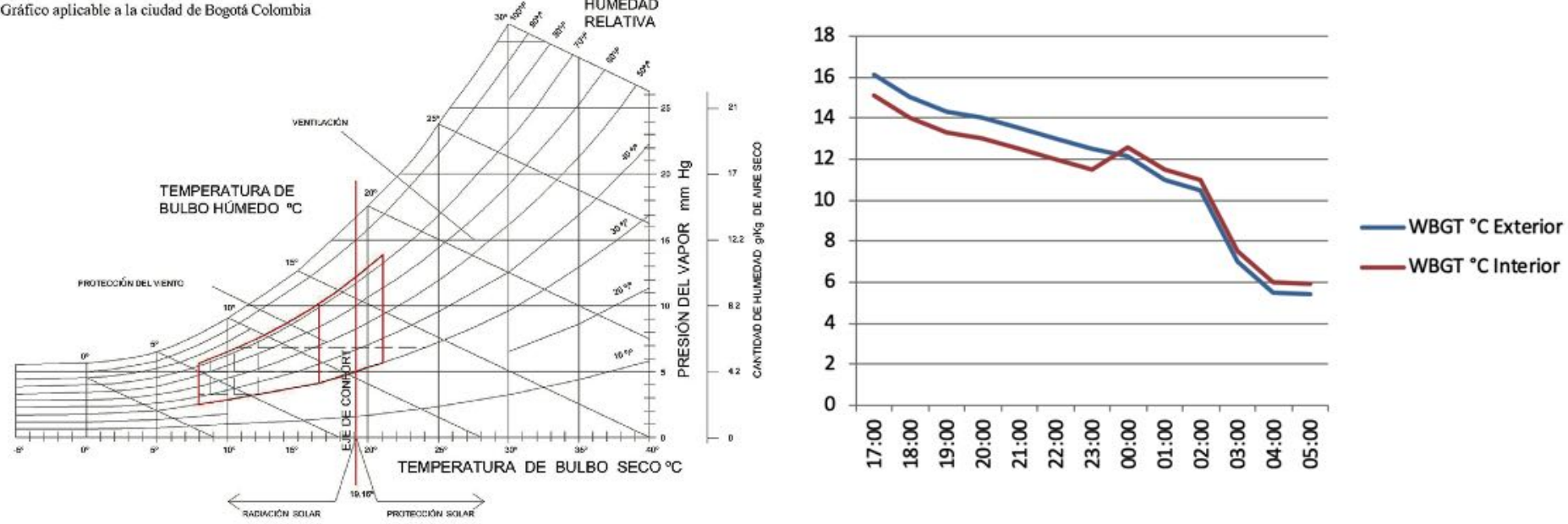

Figura 6. Gráfico de Givoni. Fuente: Elaboración del autor. 
15 y $25 \%$ en fachadas (Figura 5). A partir de este análisis inicial, el proyecto se enfocó en la cubierta de la vivienda, teniendo en cuenta los materiales y el análisis del descenso de la temperatura interior (Figura 7).

\section{LA CUBIERTA}

Con el fin de incrementar el confort térmico del hábitat de una forma sostenible, se llevó a cabo una caracterización de los materiales usados en la construcción existente considerando su densidad, calor específico, conductividad térmica y difusión térmica (Figura 9). Tomando como base los datos obtenidos y también las variables "economía", "sostenibilidad" y "conductividad térmica", se seleccionaron los materiales más viables, con el fin de mitigar las falencias detectadas en el estudio térmico realizado (Figura 10).

Aunque el fibrocemento y el Tetrapak tenían precios similares, se encontraron otros aspectos favorables para usar este último: leve disminución en la conductividad térmica y baja contaminación generada por un material que es producto del reciclaje. No obstante, se comprobó que, si se usaba solamente una teja de este material, no se obtenía el aislamiento térmico necesario, por lo que, además de las tejas exteriores, se usaron láminas Tetrapak para conformar un falso techo que le permitiera al hábitat tener un cámara de aire entre los dos elementos (Figura 10).

Para responder a las exigencias de los usuarios, se tomó la decisión de cambiar la cubierta actual construida en zinc, sobre una estructura de madera en mal estado. La nueva cubierta se construyó en Tetrapak, usando las vigas en madera existentes -que fueron limpiadas y reforzadas-, con un falso techo de láminas, cuyo espesor fue de $2 \mathrm{~cm}$ y fueron fabricadas con el mismo material, con el fin de crear un aislamiento que mitigara las bajas temperaturas nocturnas detectadas mediante el análisis climático de la zona. Se agregaron como aislantes térmicos cubos de heno con una altura de $30 \mathrm{~cm}$; material que fue seleccionado por su baja

\begin{tabular}{|c|c|c|}
\hline Material & $\begin{array}{c}\text { Densidad } \\
\left(\mathbf{k g} / \mathbf{m}^{3}\right)\end{array}$ & $\begin{array}{c}\text { Conductividad } \\
\text { térmica(W/(m/K)) }\end{array}$ \\
\hline Teja de zinc & 7140 & $106-140$ \\
\hline $\begin{array}{c}\text { Ladrillo de } \\
\text { mampostería }\end{array}$ & 1700 & 0,658 \\
\hline Madera de pino & 650 & 0,163 \\
\hline Vidrio & 2700 & 0,81 \\
\hline $\begin{array}{c}\text { Tejas de } \\
\text { fibrocemento }\end{array}$ & 1250 & 0,36 \\
\hline
\end{tabular}

Figura 8. Caracterización de los materiales de usados en la construcción de la vivienda. Fuente: Elaboración del autor.

\begin{tabular}{|c|c|c|c|c|}
\hline Descripción & $\begin{array}{c}\text { Densidad } \\
\mathbf{k g} / \mathbf{m}^{3}\end{array}$ & $\begin{array}{c}\text { Conductividad } \\
\text { térmica } \\
\mathbf{W} / \mathbf{m}^{2}{ }^{\circ} \mathbf{C}\end{array}$ & $\begin{array}{c}\text { Precio } \\
\text { USD }\end{array}$ & $\begin{array}{c}\text { Huella } \\
\text { de } \\
\text { carbono } \\
\mathbf{k g} / \mathbf{C O}^{2}\end{array}$ \\
\hline Fibrocemento & 1250 & 0,36 & $\$ 9$ & 11,84 \\
\hline $\begin{array}{c}\text { Panel tipo } \\
\text { sándwich }\end{array}$ & 50 & 0,024 & $\$ 28$ & 12,5 \\
\hline Poliéster & 38 & 0,04 & $\$ 15$ & 15,04 \\
\hline Tetrapak & 900 & 0,25 & $\$ 8$ & 0,5 \\
\hline
\end{tabular}

Figura 9. Caracterización de los materiales que mejoran el aislamiento térmico. Fuente: Elaboración del autor.

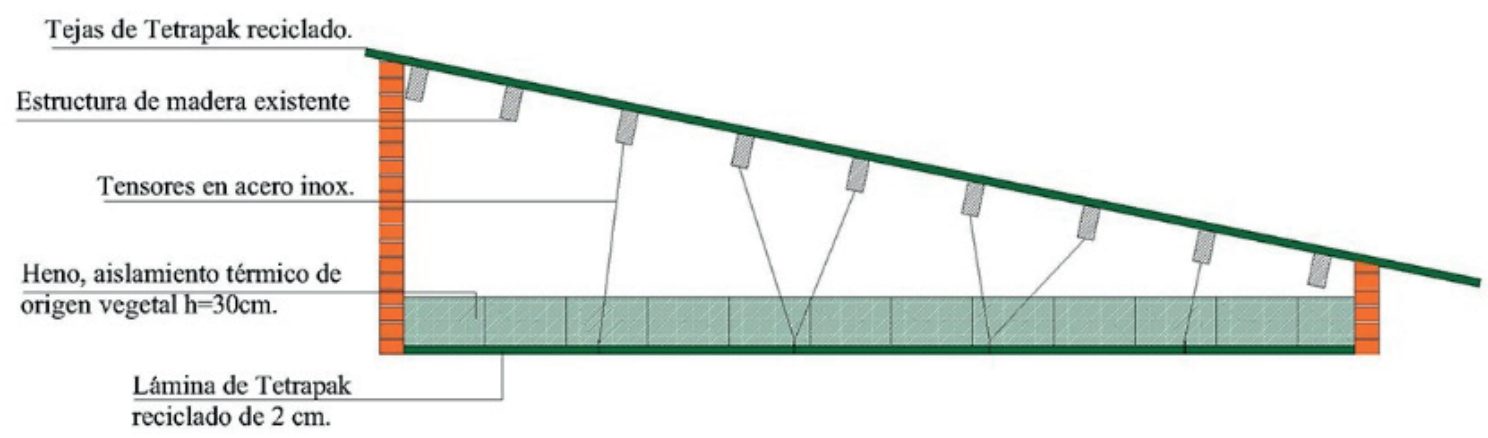

Figura 10. Sección de la nueva cubierta propuesta. Fuente: Elaboración del autor. 


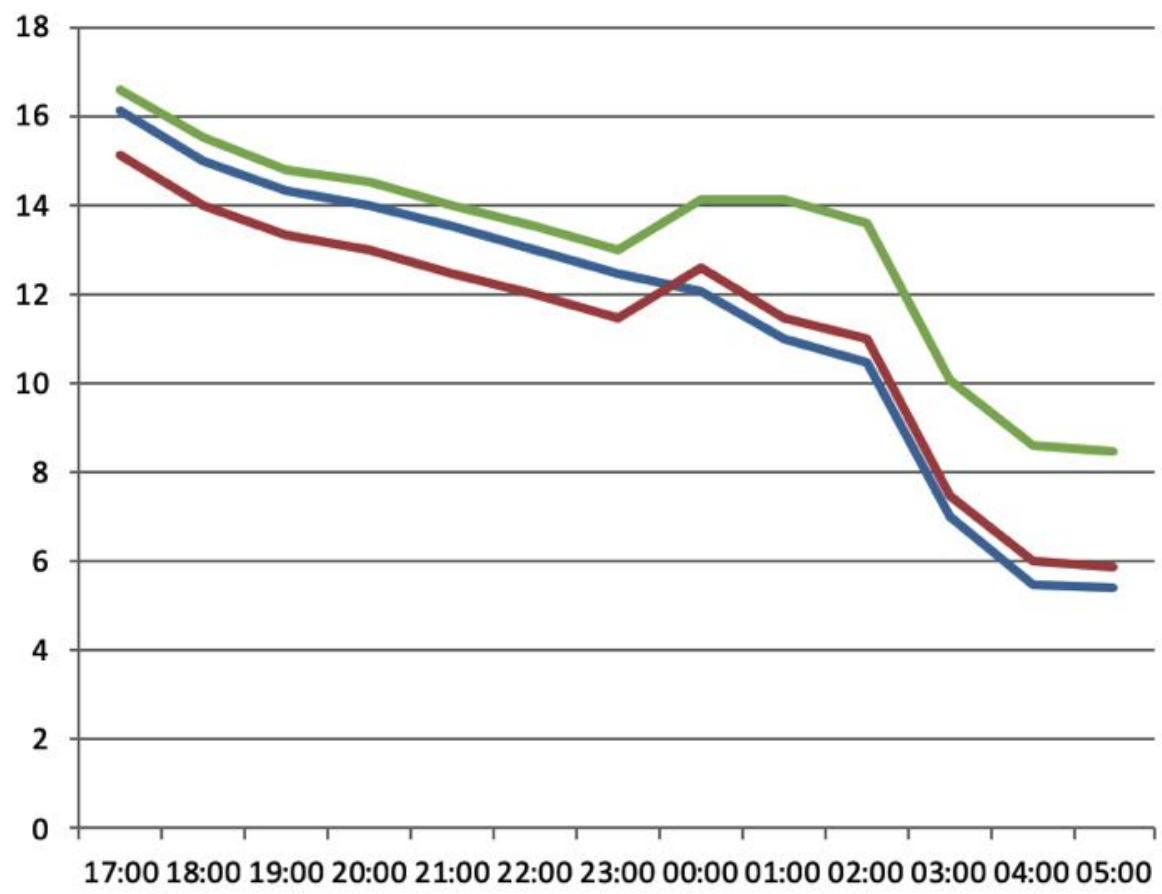

Exterior Interior antes Interior después

Figura 11. Temperatura media antes y después de la solución propuesta. Fuente: Elaboración de los autores.

\begin{tabular}{|c|c|c|}
\hline Material & Precio/m² & $\mathrm{CO}^{2} \mathrm{~kg}$ \\
\hline Tejas en fibro-cemento & $\$ 9$ & 11,85 \\
\hline Tejas en Tetrapak & $\$ 8$ & 0.5 \\
\hline
\end{tabular}

Figura 12. Fibro-cemento y Tetrapak. Precio y $\mathrm{CO}^{2}$. Fuente: ITEC (www.itec.cat.).

conductividad térmica, su bajo precio y su origen vegetal, lo que conlleva una carga muy baja por emisiones de $\mathrm{CO}^{2}$. El uso de materiales sostenibles está directamente relacionado con su disponibilidad geográfica, una de las variables tenidas en cuenta para el cálculo de la huella de carbono de cualquier material (Sekhar y Nayak, 2018).

\section{RESULTADOS}

En este punto, se comprobó el aumento de la temperatura en el interior del hábitat y se hicieron nuevas mediciones para determinar la temperatura media, la humedad y el índice de estrés térmico. Asimismo, se compararon las mediciones antes y después de la incorporación de la nueva cubierta, observándose que la diferencia entre la temperatura exterior e interior se amplió en 6 grados, de manera que en la madrugada se consiguió llegar a 13 grados en promedio al interior del hábitat (Figura 11).

El análisis de las mediciones climatológicas arrojó los siguientes resultados: el hábitat seleccionado no presentaba confort térmico en la noche ni en la madrugada; la inercia térmica de los materiales empleados en su construcción era muy baja; los materiales reutilizados tanto en la fachada como en la cubierta (láminas, zinc y tejas de asbesto cemento, que tienen una inercia térmica de 0,8 (W/ $(\mathrm{m} \cdot \mathrm{K})$ ), no contribuían a optimizar la inercia térmica. Por lo tanto, se determinó que era necesario sustituir estos cerramientos temporales empleados en la construcción de la cubierta de la vivienda, los cuales afectaban el confort térmico del hábitat analizado (Figura 7).

Los análisis climatológicos en los que se hicieron mediciones de temperatura y humedad permitieron 
determinar el índice WBGT (Wet Bulb Globe Temperature) de estrés térmico y establecer que la diferencia entre la temperatura externa e interna oscilaba entre uno y medio grado centígrados. Ahora bien, la temperatura en esta zona de Bogotá entre las 3 y 5 de la madrugada es cercana a los 5 grados, es decir, los usuarios estaban realmente expuestos a temperaturas muy bajas y fuera de la temperatura operativa de confort térmico $\left(17,21^{\circ} \mathrm{C}\right)$, las cuales ciertamente se acercaban a aquellas que soporta un habitante de la calle en esta ciudad (Figura 11). Con todo, se detectó un incremento de $1{ }^{\circ} \mathrm{C}$ después de las 23:00 debido al uso de electrodomésticos, principalmente la televisión.

Este estudio pudo establecer que es posible aumentar el confort térmico de un hábitat informal utilizando materiales sostenibles, lo que puede ser un modelo a seguir en los planes de mejoramiento de barrios, planteados por el gobierno local, esto es, sustituyendo un procedimiento en el que siempre se han empleado materiales altamente contaminantes, como el cemento y el acero, por uno en el que se utilicen otros materiales generados a partir del reciclaje.

Este proyecto demostró que es posible construir un hábitat sustentable (al usar materiales reciclados y limitar el uso del cemento) sin afectar el presupuesto destinado para su construcción y, a su vez, mejorar el confort térmico. Este hallazgo puede ser implementado en las políticas actuales de mejoramiento de vivienda planteas desde los años ochenta y en donde la sustentabilidad no era una variable tenida en cuenta para la elaboración de la norma. Se calculó que el uso del Tetrapak en este mejoramiento permitió disminuir las emisiones de $\mathrm{CO}^{2}$ en un $80 \%$ (Figura 12).

\section{DISCUSIÓN.}

Es necesario remarcar que todos los estudios de confort térmico latinoamericanos ponen especial énfasis en encontrar soluciones económicas que mitiguen las condiciones climáticas, con el principal objetivo de reducir el uso de sistemas de climatización, dado los altos costos de instalación, mantenimiento y el alto consumo eléctrico asociado a su uso. Sin embargo, se debe aclarar que la sustentabilidad de este objetivo no es evidente para la población. Es muy interesante, en ese sentido, encontrar estudios como el de Macias et al. (2015), que evaluó la reflectancia solar de los techos de viviendas en Guayaquil, Ecuador, con el fin de aminorar el uso de sistemas tradicionales de climatización, y concluyó que el uso de la chapa de acero disminuía la temperatura interior gracias al aumento de la reflectancia.

Aunque se pudo determinar que la variable económica no es la única en juego, algunas de las investigaciones latinoamericanas relacionadas con el confort se han centrado en otros aspectos, entre ellos: la percepción psicológica en Brasil (Silveira Hirashima, de Assis y Nikolopoulou, 2016), casos de estudio que analizan la relación entre la iluminación y la hidrometría en Argentina (Boutet, Hernández y Jacobo, 2020), y una propuesta para un nuevo modelo de confort adaptativo en Chile, que busca adaptar el límite inferior del umbral de confort térmico con el fin de desarrollar un estándar que refleje mejor las necesidades de los habitantes y la cultura socioeconómica (PérezFargallo et al., 2018)including adaptation to external temperatures, opening windows and changing clothing. In this regard, two international standards provide the fundamental basis to model the necessary equations: EN 16798 (formerly 15251. Así también cabe destacar el trabajo de Castillo y Huelsz (2017) donde se determinó que el confort térmico producido cuando hay ventilación natural en climas cálidos en Méjico define estrategias bioclimáticas para espacios urbanos existentes, basadas en componentes morfotipológicos, condiciones de microclima urbano y requisitos de confort para todo tipo de ciudadanos en Méjico y España.

La definición de confort térmica especifica que: "El consumo de energía o temperatura interior de un espacio determinado, bajo ciertas cargas, se puede establecer considerando estándares de confort térmico; por lo tanto, sigue siendo crucial definir adecuadamente esos estándares para lograr la comodidad de los usuarios y reducir el consumo de energía" (Pérez-Fargallo et al., 2018, p. 95). Es decir, se trata de neutralizar o evitar las condiciones climáticas dañinas y potenciar las buenas en relación a la comodidad de los usuarios.

Los primeros estudios e investigaciones sobre el confort térmico se centraban inicialmente en el uso de la ropa en las estaciones de invierno y verano (Heathcote, 2011). Esta metodología se llevó a la arquitectura, al acondicionamiento del espacio, a las condiciones climáticas: aire acondicionado, ventilación, apertura de las ventanas, aislamientos, entre otras medidas.

Hay que indicar que en los países latinoamericanos ubicados en la zona del trópico no es habitual implementar sistemas de calefacción ni aire acondicionado; este tipo de sistemas es solamente utilizado por grandes empresas y cadenas hoteleras.

Concretamente, en la vivienda informal analizada, los materiales empleados eran de carácter temporal, con el objetivo de protegerse simplemente de la intemperie; el propietario del hábitat no era consciente de los peligros asociados al uso de materiales prohibidos en otros países, como el asbesto cemento. En Colombia, la normativa que lo prohíbe desde 2019. 


\section{CONCLUSIONES}

El hábitat estudiado en la investigación aquí expuesta presentaba deficiencias en su proceso constructivo debido al carácter temporal de los materiales usados para su construcción. El objetivo principal del trabajo, incrementar el confort térmico de dicho hábitat, se cumplió parcialmente, porque el único el elemento constructivo que se pudo optimizar fue la cubierta, a causa de limitaciones de tiempo y de dinero propias del proyecto.

Sin embargo, el uso de materiales reciclados no es un aspecto inédito en investigaciones enfocadas en la vivienda sostenible, según lo expone Spagnoli: "Hay que señalar que los paneles y muebles de las paredes interiores están hechos de materiales reciclados, mezclados con aglomerados, para aumentar la sostenibilidad del proyecto y reducir los gastos" (2020, p. 163).

El análisis y la caracterización de los materiales que podrían emplearse en el mejoramiento del hábitat fue la herramienta técnica que permitió justificar el uso del Tetrapak, cuyas cualidades decisivas fueron su bajo precio y las bajas emisiones de $\mathrm{CO}^{2}$ generadas en su proceso de fabricación.

A partir de este análisis, se revela la necesidad de incorporar estándares internacionales de confort térmico en la construcción de vivienda dirigida a las clases sociales más bajas, en los países donde no estén contemplados $y$, de esa forma, evitar que en los procesos de autoconstrucción, como el del presente objeto de estudio, no se considere el confort térmico ni el empleo de materiales prohibidos en otros países, como es el caso del asbesto cemento.

No todos los materiales planteados en principio pudieron ser usados en el proyecto: uno de los objetivos era implementar el uso de la tierra en hábitats informales urbanos, sin embargo, el propietario respectivo manifestó que no le interesaba, ni autorizaba su utilización, por la fragilidad del material y porque no era posible garantizar la seguridad en la fachada del hábitat.

Con todo, la incorporación de un material sostenible garantiza la sustentabilidad de este tipo de hábitats, es decir, es posible construir un hábitat sustentable usando materiales reciclados y de precios bajos.

\section{REFERENCIAS BIBLIOGRÁFICAS}

Ahmed, A., Qayoum, A. y Mir, F. Q. (2019). Investigation of the thermal behavior of the natural insulation materials for low temperature regions. Journal of Building Engineering, 26, 100849. DOI: https://doi.org/10.1016/j.jobe.2019.100849
Boutet, M. L., Hernández, A. L. y Jacobo, G. J. (2020). Methodology of quantitative analysis and diagnosis of higrothermal and lighting monitoring for school buildings in a hothumid mid-latitude climate. Renewable Energy, 145, 2463-2476. DOI: https://doi.org/10.1016/j.renene.2019.08.009

Castillo, J. A. y Huelsz, G. (2017). A methodology to evaluate the indoor natural ventilation in hot climates: Heat Balance Index. Building and Environment, 114, 366-373. DOI: https://doi. org/10.1016/j.buildenv.2016.12.027

Chowdhury, D. y Neogi, S. (2019). Thermal performance evaluation of traditional walls and roof used in tropical climate using guarded hot box. Construction and Building Materials, 218, 73-89. DOI: https://doi.org/10.1016/j.conbuildmat.2019.05.032

Da Silveira Hirashima, S. Q., de Assis, E. S. y Nikolopoulou, M. (2016). Daytime thermal comfort in urban spaces: A field study in Brazil. Building and Environment, 107, 245-253. DOI: https://doi. org/10.1016/j.buildenv.2016.08.006

Fernández-Agüera, J., Domínguez-Amarillo, S., Alonso, C. y Martín-Consuegra, F. (2019). Thermal comfort and indoor air quality in low-income housing in Spain: The influence of airtightness and occupant behaviour. Energy and Buildings, 199, 102-114. DOI: https://doi.org/10.1016/j.enbuild.2019.06.052

Godoy Muñoz, A. de J. (2012). El confort térmico adaptativo. Aplicación en la edificación en España. Tesis de Maestría. Universitat Politècnica de Catalunya. Recuperado de https:// upcommons.upc.edu/handle/2099.1/18763

Heathcote, K. (2011). El comportamiento térmico de los edificios de tierra. Informes de La Construcción, 63(523), 117-126. DOI: https://doi.org/10.3989/ic.10.024

IDEAM (2007). Portal GeoNetwork para datos espaciales e información. Sistema de Información Ambiental del Instituto de Hidrología, Meteorología y Estudios Ambientales (IDEAM). Recuperado de http://geoservicios.ideam.gov.co:8080/ geonetwork/srv/spa/catalog.search;jsessionid=07

58FF83B771AFADEBCB46A817AD9B67\#/search?facet. $\mathrm{q}=$ topicCat\%2FclimatologyMeteorologyAtmosphere

Jones, R. V., Goodhew, S. y de Wilde, P. (2016). Measured Indoor Temperatures, Thermal Comfort and Overheating Risk: Post-occupancy Evaluation of Low Energy Houses in the UK. Energy Procedia, 88, 714-720. DOI: https://doi.org/10.1016/j. egypro.2016.06.049

Macias, J., Soriano, G., Sánchez, H. y Canchingre, Y. (2015). Assessment of solar reflectance of roofing assemblies of dwellings in Guayaquil, Ecuador. WIT Transaction on Ecology and The Environment, 195, 307-315. DOI: https://doi.org/10.2495/ ESUS150261

Pérez-Fargallo, A., Pulido-Arcas, J. A., Rubio-Bellido, C., Trebilcock, M., Piderit, B. y Attia, S. (2018). Development of a new adaptive comfort model for low income housing in the centralsouth of chile. Energy and Buildings, 178, 94-106. DOI: https:// doi.org/10.1016/J.ENBUILD.2018.08.030

Robledo-Fava, R., Hernández-Luna, M. C., Fernández-deCórdoba, P., Michinel, H., Zaragoza, S., Castillo-Guzman, A. y Selvas-Aguilar, R. (2019). Analysis of the Influence Subjective Human Parameters in the Calculation of Thermal Comfort and 
Energy Consumption of Buildings. Energies, 12(8), 1531. DOI: https://doi.org/10.3390/en12081531

Sekhar, D. y Nayak, S. (2018). Utilization of granulated blast furnace slag and cement in the manufacture of compressed stabilized earth blocks. Construction and Building Materials, 166, 531-536. DOI: https://doi.org/10.1016/J.CONBUILDMAT.2018.01.125

Spagnoli, F. (2020). A New Inclusive Housing Prototype. En International Conference on Applied Human Factors and Ergonomics (pp. 163-175). Springer, Cham. DOI: https://doi. org/10.1007/978-3-030-20444-0_16 